\title{
Exchange interaction effects in the thermodynamic properties of quantum dots
}

\author{
L. G. G. V. Dias da Silva ${ }^{1,2, *}$ and Nelson Studart ${ }^{2}$ \\ ${ }^{1}$ Department of Physics and Astronomy, Nanoscale and Quantum \\ Phenomena Institute, Ohio University, Athens, Ohio 45701-2979 \\ ${ }^{2}$ Departamento de Física, Universidade Federal de São Carlos, 13565-905 São Carlos SP, Brazil
}

(Dated: February 8, 2020)

\begin{abstract}
We study electron-electron interaction effects in the thermodynamic properties of quantum-dot systems. We obtain the direct and exchange contributions to the specific heat $C_{v}$ in the selfconsistent Hartree-Fock approximation at finite temperatures. An exchange-induced phase transition is observed and the transition temperature is shown to be inversely proportional to the size of the system. The exchange contribution to $C_{v}$ dominates over the direct and kinetic contributions in the intermediate regime of interaction strength $\left(r_{s} \sim 1\right)$. Furthermore, the electron-electron interaction modifies both the amplitude and the period of magnetic field induced oscillations in $C_{v}$.
\end{abstract}

PACS numbers: 73.21.La, 21.60.Jz 65.80.+n,

\section{INTRODUCTION}

Research on semiconductor quantum dots (QD)s and nanostructures have drawn considerable effort in recent years $\stackrel{1}{\underline{1}}$ In particular, the study of electron-electron interaction effects on the ground-state and excited-state of QDs has been a very active subject. A variety of methods have been used in such studies, ranging from the exact diagonalization of few electron systems 2.3 .4 .5 .6 .7 to sophisticated numerical schemes based on the density functional theory, quantum Monte Carlo simulations and mean-field approximations $\underline{8}^{-}$Among the last ones, the self-consistent Hartree-Fock (SCHF) approximation has been successfully applied to QDs in a number of works 9.10.11.12.13.14.15.16 which focused attention on calculations of the pair-correlation function $\frac{9}{}$ and addition spectra ${ }^{10.11}$ and on configurations of the Wigner-like molecule in the strong interacting regime ${ }^{12.13}$

A less pursued track is the use of SCHF to study magnetic and thermodynamic properties of semiconductor QDs ${ }^{14.15 .16}$ Electron-electron interaction was shown to give an important contribution to thermodynamic properties such as the magnetization ${ }^{7.14}$ and the magnetic susceptibility ${ }^{6.16}$ Another quantity of experimental interest is the specific heat $C_{v}$, which has been studied in a number of works in both non-interacting 17.18 and interacting, 2,15 QD systems. In Ref. 15, Dean and coworkers reported an interesting interaction-induced phase transition in parabolic QDs with $N \sim 6$ electrons. This phase transition manifests itself as sharp drops in the specific heat as the temperature reaches a critical value. Nevertheless, a systematic study on how such a transition depends on the interaction coupling parameter $r_{s}$ of the dot, which measures the relative electronelectron interaction strength, remains to be performed.

In this paper, we address the role of the exchange interaction in the thermodynamic properties of non-parabolic QDs. Specifically, we study the kinetic, direct and exchange contribution to the specific heat in a finitetemperature Hartree-Fock approach ${ }^{10.11}$ In a previous work using this method, ${ }^{16}$ we have shown that the ex- change interaction contribution is the dominant term in magnetic properties such as the zero-field susceptibility in the intermediate regime of interaction strength $\left(r_{s} \sim 1\right)$. We find in this investigation that the exchange effects also play a dominant role on the specific heat properties. In particular, the exchange electron correlations dominate the finite-temperature phase transition and is the leading contribution to $C_{v}$ for $r_{s} \sim 1$. We also find that the transition temperature scales with the inverse of the dot size. As a consequence, this phase transition could in principle be experimentally observed for dots tens of nanometers across at an attainable temperature range.

The paper is organized as follows. In Section [II we describe the system to be studied and its main features. The results for the specific heat and the discussion of main results are given in Section remarks.

\section{THE MODEL}

We consider the problem of $N$ interacting electrons confined in a $2 \mathrm{D}$ square quantum dot of size $L$ and subjected to an external magnetic field $\mathbf{B}$ perpendicular to the electron system. To account for screening effects, the electron-electron interaction is modeled by an Yukawatype potential ${ }^{6}$ and the model Hamiltonian reads as

$$
H=\sum_{n=1}^{N} h\left(\mathbf{r}_{n}\right)+\sum_{n<n^{\prime}}^{N} \frac{e^{2}}{\epsilon_{r}} \frac{e^{-\kappa\left|\mathbf{r}_{n}-\mathbf{r}_{n^{\prime}}\right|}}{\left|\mathbf{r}_{n}-\mathbf{r}_{n^{\prime}}\right|},
$$

where $\mathbf{r}_{n}$ indicates the position of the $n$th electron. We consider low $g$-factor QDs, so that the Zeeman term can be safely disregarded. Above, $\kappa$ gives the effective interaction range and $\epsilon_{r}$ is the background dielectric constant. For $\kappa=0$, there are no screening effects and the "bare" Coulomb interaction is recovered.

The single-particle Hamiltonian $h(\mathbf{r})$ is given by

$$
h(\mathbf{r})=\frac{1}{2 m^{*}}\left[\mathbf{p}+\frac{e}{c} \mathbf{A}(\mathbf{r})\right]^{2}+u(\mathbf{r}),
$$


where $m^{*}$ is the electron effective mass and $u(\mathbf{r})$ is the hard-wall confining potential. The vector potential $\mathbf{A}$ is chosen in the symmetric gauge, namely, $\mathbf{A}=$ $(-B y / 2, B x / 2,0)$. Hereafter, the magnetic field is expressed in units of $\Phi / \Phi_{0}$, where $\Phi=B \mathcal{A}$ is the magnetic flux through the system area $\mathcal{A}$ and $\Phi_{0}=h c / e$ is the quantum flux unit.

A key parameter in our analysis is $L / a_{B}^{*}$, the $\mathrm{QD}$ length $L$ in units of the effective Bohr radius $a_{B}^{*}=$ $\hbar^{2} \epsilon_{r} / m^{*} e^{2}$ which gives the relative strength of the ee interaction as compared to the kinetic energy of the system .10 For a square dot of side $L$, the potential energy scales with $L^{-1}$ while the kinetic energy scales with $L^{-2}$. Therefore, as $L$ is increased, the potential energy becomes increasingly more important.

The standard dimensionless parameter that quantifies the ratio between the potential and kinetic energies of the system is the so-called Brueckner parameter $r_{s}$, which in $2 \mathrm{D}$ reads as $r_{s}^{2}=\mathcal{A} /\left(N \pi\left[a_{B}^{*}\right]^{2}\right)$. Therefore, $L / a_{B}^{*}$ and $r_{s}$ are related by $r_{s}=\left(L / a_{B}^{*}\right) / \sqrt{\pi N}$. Furthermore, by choosing a square hard-wall confinement, one can easily tune $r_{s}$ by changing the dot's lateral size $L$.

The many-body ground-state energy is obtained in the finite-temperature SCHF approximation. The SCHF equations read as 11.15

$$
\begin{array}{r}
h(\mathbf{r}) \phi_{i}(\mathbf{r})+\sum_{j}\left[n_{j} \int d \mathbf{r}^{\prime} \phi_{j}^{*}\left(\mathbf{r}^{\prime}\right) v\left(\mathbf{r}, \mathbf{r}^{\prime}\right) \phi_{j}\left(\mathbf{r}^{\prime}\right)\right] \phi_{i}(\mathbf{r}) \\
-\sum_{j}\left[n_{j} \int d \mathbf{r}^{\prime} \phi_{j}^{*}\left(\mathbf{r}^{\prime}\right) v\left(\mathbf{r}, \mathbf{r}^{\prime}\right) \phi_{j}(\mathbf{r}) \phi_{i}\left(\mathbf{r}^{\prime}\right)\right]=\varepsilon_{i}^{\mathrm{HF}} \phi_{i}(\mathbf{r}),
\end{array}
$$

where the sums run over all HF orbitals. Here $n_{i}=$ $\left\{\exp \left[\left(\varepsilon_{i}^{\mathrm{HF}}-\mu\right) / k_{B} T\right]+1\right\}^{-1}$ is the Fermi occupation number of the $i$ th HF orbital with corresponding wave function $\phi_{i}(\mathbf{r})$ and energy $\varepsilon_{i}^{\mathrm{HF}}$. As in the standard procedure, the chemical potential $\mu$ is determined by requiring that $N=\sum_{i} n_{i}$. We truncate the number of orbitals and take only the $M \geq 2 N$ lowest energy states into account.

The SCHF ground-state energy is given by

$$
\begin{aligned}
E_{g}^{\mathrm{HF}} \equiv & T^{\mathrm{HF}}+V_{d}^{\mathrm{HF}}-V_{x}^{\mathrm{HF}} \\
= & \sum_{i} n_{i}\left\langle\phi_{i}|h| \phi_{i}\right\rangle+\frac{1}{2} \sum_{i, j} n_{i} n_{j}\left(\left\langle\phi_{i} \phi_{j}|v| \phi_{i} \phi_{j}\right\rangle\right. \\
& \left.-\left\langle\phi_{i} \phi_{j}|v| \phi_{j} \phi_{i}\right\rangle\right),
\end{aligned}
$$

where the $\left|\phi_{i}\right\rangle$ are the HF orbitals, self-consistent solutions of Eq. (3) and $T^{\mathrm{HF}}, V_{d}^{\mathrm{HF}}$ and $V_{x}^{\mathrm{HF}}$ are the kinetic, direct and exchange contributions to the ground-state energy respectively. We are interested in the intermediate interaction strength regime $\left(r_{s} \sim 1\right)$. In this regime, the direct interaction term $V_{d}^{\mathrm{HF}}$ is the leading contribution to the $E_{g}^{\mathrm{HF}}$, followed by $T^{\mathrm{HF}}$ and $V_{x}^{\mathrm{HF}}$ respectively.

The details on the matrix elements calculations and the Hartree-Fock method can be found on Refs. [6 and 16 respectively.

We calculate the specific heat $C_{v}$ of the system in this SCHF approximation, namely:

$$
C_{v}=\left(\frac{\partial U}{\partial T}\right)_{V}
$$

where $U$ is the internal energy and $T$ is the system temperature. The first-order exchange and Hartree contributions to $C_{v}$ can be accounted for by approximating $U \approx E_{g}^{\mathrm{HF}}$ so that for $E_{g}^{\mathrm{HF}}$, given by Eq. [4 there are kinetic $\left(C_{v}^{\mathrm{kin}}\right)$, direct $\left(C_{v}^{d}\right)$ and exchange $\left(C_{v}^{x}\right)$ contributions to $C_{v}$.

\section{RESULTS AND CONCLUSIONS}

We analyze the behavior of the specific heat $C_{v}$ as a function of relevant parameters of the system, i.e. the temperature $T$, the interaction strength $L / a_{B}^{*}$, the magnetic field $\Phi / \Phi_{0}$, the potential range $\kappa^{-1}$ and the number of electrons $N$. The results given in this section are for the Coulomb case $(\kappa=0)$ with $N=10$ electrons in the dot. We should mention that QDs with up to $N=20$ electrons and with the screened interaction $(\kappa \neq 0)$ were also considered and the same overall qualitative features were observed. In the remaining of this section, energy and temperature are given in units of the typical scales for the system, namely $E_{L}=\hbar^{2} /\left(m^{*} L^{2}\right)$ and $T_{L}=E_{L} / k_{B}$ respectively.

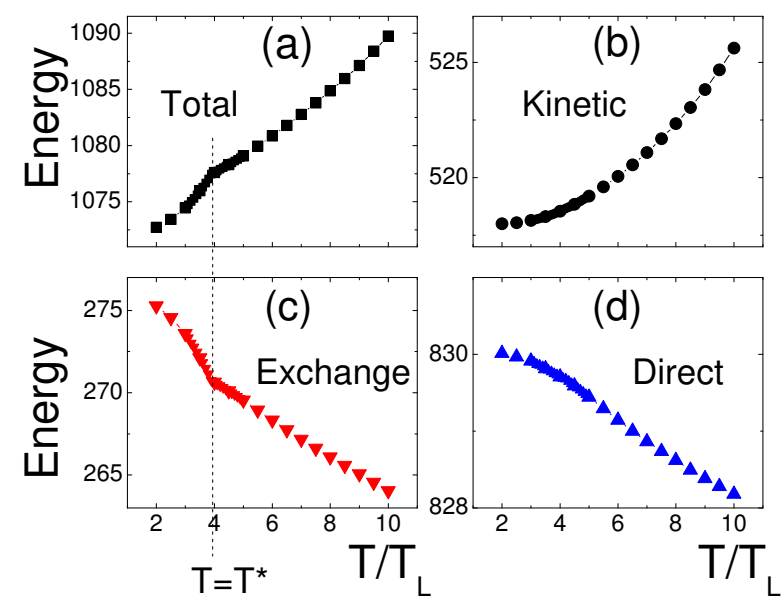

FIG. 1: (color online) (a): Ground-state energy $E_{\mathrm{HF}}$ as function of temperature for $N=10$ electrons and $r_{s}=0.89$. The kinetic (b), exchange (c) and direct (d) contributions to $E_{\mathrm{HF}}$ are also plotted.

The ground-state energy $E_{g}^{\mathrm{HF}}$ increases with temperature as shown in Fig. In for $N=10$ and $r_{s}=0.89$. Nevertheless, this increase is not smooth and a sudden change in slope is observed at a certain temperature $T^{*}$. By analyzing the kinetic energy $T^{\mathrm{HF}}$, the direct term $V_{d}^{\mathrm{HF}}$ and the exchange contribution $V_{x}^{\mathrm{HF}}$ given by Eq. 


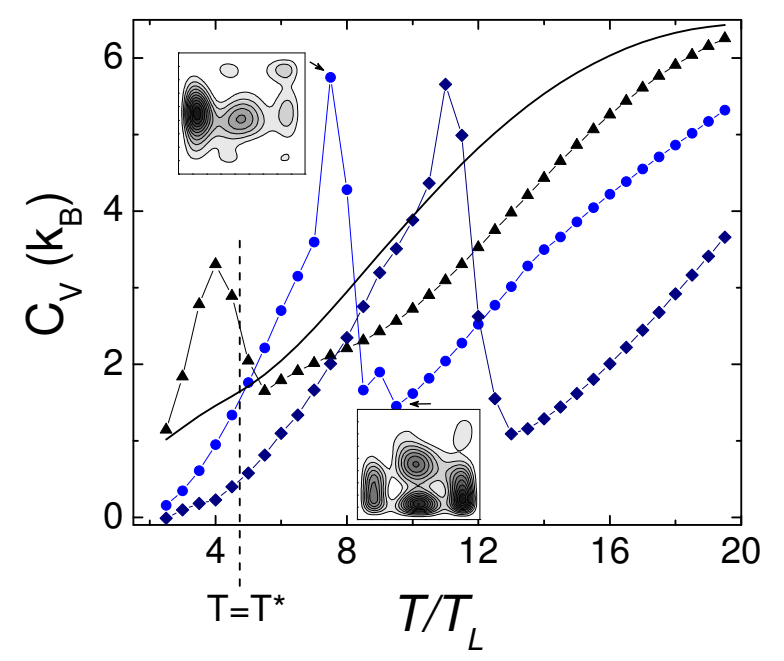

FIG. 2: (color online) Specific heat as function of temperature for the noninteracting (solid line) and interacting cases. In the latter, the interaction strength values are $r_{s}=1.07$ (up triangles), 2.14 (circles) and 3.30 (diamonds). The insets show typical charge distributions in the dot before and after the transition.

(4), we see that both $V_{d}^{\mathrm{HF}}$ and $V_{x}^{\mathrm{HF}}$ decrease with $T$, as expected. Furthermore, we observe clearly that the change in slope is a feature due to the exchange interaction, since neither $T^{\mathrm{HF}}$ or $V_{d}^{\mathrm{HF}}$ display cusps at $T=T^{*}$ (Figs. 1b-d).

The abrupt change in slope in the energy causes a discontinuity in the specific heat $C_{v}(T)$ at $T=T^{*}$, as seen in Fig. 2] A sharp drop develops for a wide range of values of the interaction strength parameter (for simplicity, the noninteracting case is referred to as " $L / a_{B}^{*}=0$ " or " $r_{s}=0 "$. The noninteracting curve does not display any sharp drops).

Discontinuities in the specific heat are usually regarded as signatures of phase transitions $\frac{19}{19}$ In fact, such transitions are accompanied by a charge reordering in the ground state distribution, as shown in the insets of Fig. 2 The lack of the $\mathcal{C}_{4}$ rotational symmetry in the charge distributions is a consequence of the nonlinear coupling of the original orbitals in the Eq. (3) and is a peculiarity of the Hartree-Fock approximation. Nonetheless, a clear charge rearrangement is verified as the system undergoes the phase transition. Most strikingly is the fact that this is an exchange-induced phase transition and it is a direct consequence of exchange effects between the electrons in the dot. Such transitions were reported in previous studies $\frac{15}{5}$ as related to phase transitions in the ground-state charge distribution inside the dot.

The reduced transition temperature varies both as a function of the number of electrons and the relative interaction strength. For $N=20$, up to three transitions are observed in the temperature range $2<T / T_{L}<20$ (not

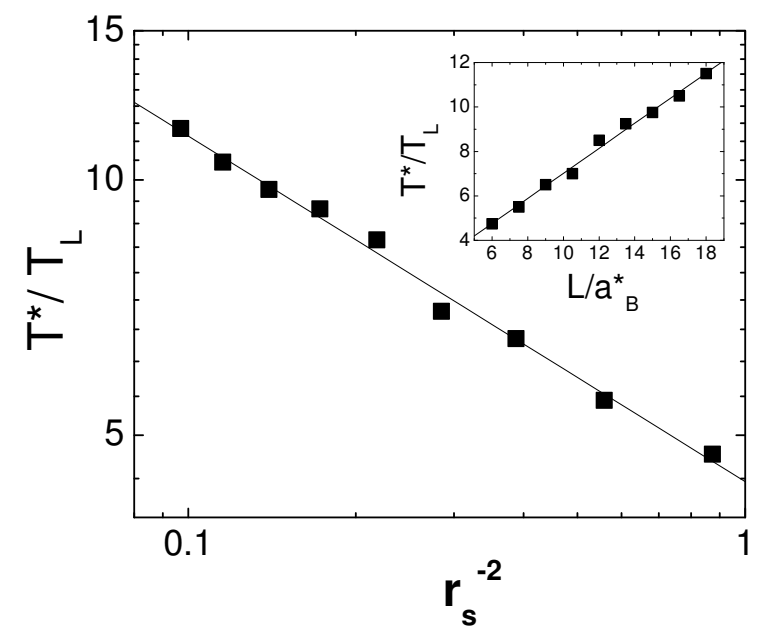

FIG. 3: Reduced transition temperature $T^{*} / T_{L}$ as function of $r_{s}^{-2}$ (see text for details). Inset: $T^{*} / T_{L}$ increases linearly with the interaction strength $L / a_{B}^{*}$.

shown). In Fig. 3 the dependence of the critical temperature $T^{*} / T_{L}$ with $r_{s}^{-2}$ (which is proportional to the density $N / L^{2}$ ) is shown. We also depict $T^{*} / T_{L}$ as a function of $L / a_{B}^{*}$ in the inset of Fig. [3] and a roughly linear dependence is observed. These results imply that the transition temperature $T^{*}$ scales with $L^{-1}$ since $T_{L} \propto L^{-2}$. The data are very well described by a linear fit $\left(T^{*} \propto L^{-1}\right)$ as shown in the figure.

Such a scaling behavior allows us to estimate the values of the critical temperature $T^{*}$ for typical dot sizes. For GaAs QDs with $L \sim 50 \mathrm{~nm}$ one obtains $T^{*} \sim 11 \mathrm{~K}$ and it decreases with $1 / L$ for larger dots. The transition temperature further decreases when screening effects are taken into account. For the potential range of $\kappa^{-1}=$ $L / 10$ and $L \sim 50 \mathrm{~nm}$, we obtain $T^{*} \sim 2 \mathrm{~K}$.

We have also investigated the specific heat dependence with both the interaction strength parameter and the magnetic field for a fixed temperature. The kinetic, direct, and exchange contributions to $C_{v}$ at $T=3 T_{L}$ are shown on Fig. 4 as a function of $r_{s}$. As the relative interaction strength parameter increases, the exchange contribution $C_{v}^{x}$ rises fast and becomes the leading contribution to $C_{v}$ for $r_{s} \sim 1$. This is a surprising result since $V_{x}^{\mathrm{HF}}$ is smaller than $V_{d}^{\mathrm{HF}}$ and $T^{\mathrm{HF}}$ by a factor of $2-3$ (see Fig. 11). However, the effect of temperature in the variation of $V_{x}^{\mathrm{HF}}$ is stronger and $C_{v}^{x}>C_{v}^{\mathrm{kin}}, C_{v}^{d}$ for $r_{s} \sim 1$. The direct term, on the other hand, gives a smaller negative contribution which cancels out the positive $C_{v}^{\text {kin }}$ (which is dominant for $r_{s} \ll 1$ ). A peak in $C_{v}$ appears at the value of $r_{s}$ for which $T^{*}=3 T_{L}$ and approaches zero for higher values of $r_{s}$, since temperatures changes do not sensibly affect the ground-state energy in the strongly interacting regime.

The specific heat oscillates as a function of the mag- 


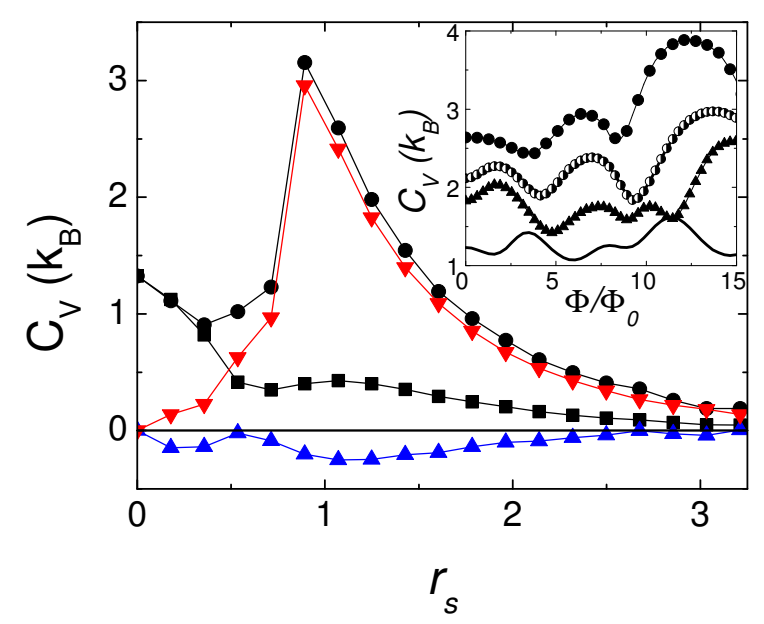

FIG. 4: (color online) Specific heat as function of $r_{s}$ (circles). Also plotted are the kinetic (squares), direct (up triangles) and exchange (down triangles) contributions. Inset: $C_{v}$ as function of magnetic flux $\Phi / \Phi_{0}$ for the noninteracting (solid line) and interacting cases with $r_{s}=0.53$ (triangles), 0.89 (half-filled circles) and 1.78 (filled circles). Curves are off-set for clarity.

netic field with increasing amplitude, as seen in the inset of Fig. 4 The interaction influences both the period and the amplitude of $C_{v}(B)$. The noninteracting curve displays an oscillatory pattern with both high and low harmonics. For higher values of the interaction strength parameter, the higher harmonics are suppressed and an oscillation period is defined more clearly . For even higher values of $r_{s}$, the oscillation period decreases. A similar behavior was seen in the magnetization and magnetic susceptibility of QD systems ${ }^{16}$ and it is related to an ef- fective increase in the chemical potential as $r_{s}$ increases.

In summary, we have investigated interaction effects in the thermodynamic properties of QDs. The exchange interaction plays a relevant role on the specific heat features and is the leading contribution for dots in the $r_{s} \sim 1$ range. The exchange induced finite-temperature

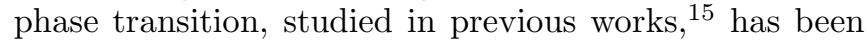
shown to depend on the interaction strength parameter, or equivalently, on the system size $L$. The transition temperature $T^{*}$ decreases as $L$ increases and we estimate that the transition regime could be experimentally accessible for sufficiently small dots. Furthermore, the specific heat oscillates with the magnetic field and both period and amplitude of such oscillations strongly depend on interaction effects.

Experiments to verify our findings using single quantum-dots are likely too demanding. The specific heat has been measured in multi-layer 2D electron gases in the Landau regime with heat-pulse ${ }^{20}$ and steady-state ac-temperature calorimetry ${ }^{21}$ techniques, with resolutions in $C_{v}$ still much lower than the required to test our results. One possible way to overcome such difficulty is to perform experiments in ensembles of nearly identical dots in a multi-layer configuration so that the contribution from single dots is amplified. This is, nonetheless, an experimentally challenging task which would bring a new understanding to the many-body effects in the thermodynamics of such small-scale devices.

\section{Acknowledgments}

We thank Profs. Caio Lewenkopf and Sergio Ulloa for helpful comments and suggestions. This work was partly supported by FAPESP (grant 01/14276-0), CNPq and NSF.
* Electronic address: gregorio@phy.ohiou.edu

1 L. L. Sohn, L. P. Kowenhoven and G. Schön, eds., Mesoscopic Electron Transport (Kluwer, New York, 1997).

2 P. A. Maksym and T. Chakraborty, Phys. Rev. Lett. 65, 108 (1990).

3 P. A. Maksym and T. Chakraborty, Phys. Rev. B 45, R1947 (1992).

4 M. Wagner, U. Merkt, and A. V. Chaplik, Phys. Rev. B 45, R1951 (1992).

${ }^{5}$ C. E. Creffield, J. H. Jefferson, S. Sarkar, and D. L. J. Tipton, Phys. Rev. B 62, 7249 (2000).

6 L. G. G. V. Dias da Silva and M. A. M. de Aguiar, Phys. Rev. B 66, 165309 (2002).

7 W. Sheng, and H. Xu, Physica B 256-258, 152-156 (1998).

8 S. M. Reimann and M. Manninen, Rev. Mod. Phys. 74, 1283 (2002).

9 D. Pfannkuche, V. Gudmundsson, and P. A. Maksym, Phys. Rev. B 47, 2244 (1993).
${ }^{10}$ K.-H. Ahn, K. Richter, and I.-H. Lee, Phys. Rev. Lett. 83, 4144 (1999).

11 H. Tamura and M. Ueda, Phys. Rev. Lett. 79, 1345 (1997).

12 Boris Reusch and Hermann Grabert, Phys. Rev. B 68, 045309 (2003).

13 B. Szafran, S. Bednarek, J. Adamowski, M.B. Tavernier, E. Anisimovas, and F.M. Peeters Eur. Phys. J. D 28, 373380 (2004).

14 G. Vasile, V. Gudmundsson, and A. Manolescu, in Proceedings of the 15th International Conference on High Magnetic Fields in Semiconductor Physics, edited by A. R. Long and J. H. Davies (IOP, Bristol, 2003), p. A11. (cond-mat/0207361)

15 D. J. Dean, M. R. Strayer, and J. C. Wells, Phys. Rev. B 64, 125305 (2001).

16 L. G. G. V. Dias da Silva, C. Lewenkopf, and N. Studart, Phys. Rev. B 69, 075311 (2004).

17 Jean-Jacques S. De Groote, J. E. M. Hornos, A. V. Chap- 
lik, Phys. Rev. B 46, 12773 (1992).

18 V. A. Geyler, V. A. Margulis, Phys. Rev. B 55, 2543 (1997).

19 H. E. Stanley, Introduction to Phase Transitions and Critical Phenomena (Clarendon Press, Oxford, 1971).
20 E. Gornik, R. Lassnig, G. Strasser, H. L. Störmer, A. C. Gossard, W. Weigmann, Phys. Rev. Lett. 54, 1820 (1985).

21 J. K. Wang, J. H. Campbell, D. C. Tsui, A. Y. Cho, Phys. Rev. B 38, 6174 (1988). 